\title{
Cumulative prospect theory for parametric and multiattribute utilities
}

Citation for published version (APA):

Zank, H. (1998). Cumulative prospect theory for parametric and multiattribute utilities. METEOR, Maastricht University School of Business and Economics. METEOR Research Memorandum No. 019 https://doi.org/10.26481/umamet.1998019

Document status and date:

Published: 01/01/1998

DOI:

10.26481/umamet.1998019

Document Version:

Publisher's PDF, also known as Version of record

\section{Please check the document version of this publication:}

- A submitted manuscript is the version of the article upon submission and before peer-review. There can be important differences between the submitted version and the official published version of record.

People interested in the research are advised to contact the author for the final version of the publication, or visit the DOI to the publisher's website.

- The final author version and the galley proof are versions of the publication after peer review.

- The final published version features the final layout of the paper including the volume, issue and page numbers.

Link to publication

\footnotetext{
General rights rights.

- You may freely distribute the URL identifying the publication in the public portal. please follow below link for the End User Agreement:

www.umlib.nl/taverne-license

Take down policy

If you believe that this document breaches copyright please contact us at:

repository@maastrichtuniversity.nl

providing details and we will investigate your claim.
}

Copyright and moral rights for the publications made accessible in the public portal are retained by the authors and/or other copyright owners and it is a condition of accessing publications that users recognise and abide by the legal requirements associated with these

- Users may download and print one copy of any publication from the public portal for the purpose of private study or research.

- You may not further distribute the material or use it for any profit-making activity or commercial gain

If the publication is distributed under the terms of Article $25 \mathrm{fa}$ of the Dutch Copyright Act, indicated by the "Taverne" license above, 


\title{
Cumulative Prospect Theory for Parametric and Multiattribute Utilities
}

\author{
Horst Zank
}

Dept. of Quantitative Economics, Maastricht University, P.O. Box 616, 6200 MD Maastricht, The Netherlands

August 17, 1998

\begin{abstract}
In cumulative prospect theory models, different behavior concerning gains and losses is permitted. For gains different decision weights are assigned than for losses, and the shape of utility can reveal loss aversion. Decision analyses concentrate on both, the capacities, which determine the decision weights, and the nature of utility. This paper focuses on linear/exponential, power and multilinear utility for decision models under uncertainty. Simple preference axioms are formulated for a representation by a cumulative prospect theory function. All models share the following axioms: weak ordering, continuity, monotonicity and tail independence. We first show that in their presence constant absolute (proportional) risk aversion implies linear/exponential (power) utility. Then, in the multiattribute case, considering (mutual) utility independence, it is shown that the utility function is (additive/multiplicative) multilinear.
\end{abstract}

Keywords: cumulative prospect theory, constant absolute (proportional) risk aversion, multiattribute utility theory. 


\section{Introduction}

Based on empirical ground cumulative prospect theory (CPT) supports the distinction of outcomes into gains and losses. The cumulative probabilities are transformed according to this separation, and the utility function also reflects that distinction. Kahneman \& Tversky (1979) and Tversky \& Kahneman (1992) proposed a utility function which is concave for gains, convex for losses, and steeper for losses than for gains. From their experiments, in which lotteries on monetary outcomes were valued, Tversky \& Kahneman (1992) concluded that a two-sided power function is a good approximate for the utility function. Different powers for gains than for losses were deduced.

The empirical analysis of Currim and Sarin (1989) confirms the properties of utility and of the weighting functions in the cumulative prospect theory model. They fitted an exponential form for utility, and found evidence for different decision weights for gains than for losses. Smidts (1997) concluded from his data, that a exponential utility fits better than a power utility. In a different experimental study Beetsma and Schotman (1998) conclude that the exponential and the power utility perform equally well.

For decision under risk a complete axiomatization of cumulative prospect theory with a two-part power utility is presented in Wakker \& Zank (1997). There, constant proportional risk aversion of the preference relation determines the nature of utility, in the presence of the simple axioms: weak ordering, continuity, stochastic dominance and tail independence, the latter being a weakening of the independence condition of von Neuman \& Morgenstern (1944).

However, as mentioned above, interest in a special form for utility is not limited to constant proportional risk aversion. Linear/exponential, additive/multiplicative or multilinear utility families are also topic for many analytic studies (Currim \& Sarin, 1989; 
Smidts 1997; Beetsma \& Schotman, 1998). Miyamoto \& Wakker (1996) derived models characterizing such families of utilities, assuming the additive representation for a preference relation on rank-ordered acts given beforehand. They point out that, when there is interest in a specific form of utility, preference axioms need not immediately imply a separation of probabilities and utilities in the representing function, but can be weakened to imply only additive representability. Then, in the presence of constant proportional (absolute) risk aversion or utility independence, probabilities and utilities can be identified. This feature will be captured in all our models. First additive representability is established, and from that a cumulative prospect theory functional is derived.

Other parametric families of utilities, focusing on decreasing or increasing risk aversion, were characterized by Farquhar \& Nakamura (1987) and Bell (1988), the so called "polynomial-exponential" utility functions, a family including the "sumex" utilities presented in Nakamura (1996). Saha (1993) proposed the "expo-power" utility, a form which exhibits decreasing or increasing absolute risk aversion and decreasing or increasing proportional risk aversion, depending on the values of the parameters involved. However, such families will not be discussed here.

This paper deals with decision under uncertainty. For a finite set of states, we first derive a CPT-model with linear or exponential utility. Here, the central property of the preference relation is constant absolute risk aversion. This, in addition to weak ordering, monotonicity, continuity and tail independence, constitutes necessary and sufficient axioms for the derivation of such a model.

Secondly, we concentrate on a CPT-model with utility as a power function, where constant proportional risk averse preferences are considered.

Thirdly, for multiattribute outcomes, we focus on preferences satisfying mutual util- 
ity independence or utility independence for attributes, deriving CPT-models with additive/multiplicative or multilinear utility, respectively. Also here the additional axioms are weak ordering, monotonicity, continuity and tail independence, simple axioms, which imply the existence of additive representing functions on rank-ordered sets.

Proofs are presented in the Appendix, except for Lemma 1.

\section{CPT with Linear/Exponential or Power Utility}

Throughout this paper $S=\{1, \ldots, n\}$, with $n \geqslant 3$, is a finite set of states, where exactly one state is true and there is uncertainty about which is the true state; subsets of $S$ are events. An act $f$ assigns to each state $j \in S$ an outcome $f(j)$, or $f_{j}$ for short. In this section the set of outcomes is the set of real numbers $I R$, and thus, we view the set of acts as the Cartesian product $\mathbb{R}^{n}$. Positive outcomes are gains and negative outcomes are losses; they are separated by the zero outcome which is the status quo. Hence, an act $f$ consists of a gain-part $f^{+}$and a loss-part $f^{-}$, where $f^{+}$is the act $f$ with all losses replaced by the status quo, and $f^{-}$is the act $f$ with all gains replaced by the status quo. Sometimes we identify the constant act $(x, \ldots, x) \in \mathbb{R}^{n}$ with the outcome $x \in \mathbb{R}$.

An act $f$ is rank-ordered if its outcomes are ordered as follows: $f_{1} \geqslant \cdots \geqslant f_{n}$. For each act there exists a permutation $\rho$ of $\{1, \ldots, n\}$ such that $f_{\rho(1)} \geqslant \cdots \geqslant f_{\rho(n)}$, i.e. the outcomes are rank-ordered with respect to $\rho$. For each permutation $\rho$ of $\{1, \ldots, n\}$ the set $I R_{\rho}^{n}$ consists of those acts which are rank-ordered according to $\rho$. For example, if $\rho=i d$ (i.e. $\rho(i)=i$ for all $i$ ), then $I R_{i d}^{n}$ is the set of rank-ordered acts.

On the set of acts we assume a preference relation denoted by $\succcurlyeq$. The symbols $\succcurlyeq, \succ$ $, \sim, \preccurlyeq, \prec$, are defined in the usual way, i.e. $f \succ g$ means $[f \succcurlyeq g$ and not $g \succcurlyeq f], f \succ g$ means $[f \succcurlyeq g$ and $g \succcurlyeq f], f \preccurlyeq g$ means $g \succcurlyeq f$, and $f \prec g$ means $g \succ f$. 
$V$ is a representing function or representation for $\succcurlyeq$ if $V$ assigns to each act a real value such that $f \succcurlyeq g \Leftrightarrow V(f) \geqslant V(g)$. If such a representing function exists then $\succcurlyeq$ is a weak order, i.e. $\succcurlyeq$ is complete $(f \succcurlyeq g$ or $g \succcurlyeq f$ for all acts $f, g)$ and transitive.

One of the best known representations is subjective expected utility (SEU). It holds whenever the representing function has the following form:

$$
\left(f_{1}, \ldots, f_{n}\right) \mapsto i=1 \sum^{n} p_{i} \cdot U\left(f_{i}\right)
$$

The subjective probabilities $p_{i}$, for $i=1, \ldots, n$ are uniquely determined; they are nonnegative and their sum equals 1 . The utility function $U$ maps from the set of outcomes into the reals. In this paper we consider only continuous and strictly increasing utility functions. In the above formula $U$ is cardinal, i.e. unique up to a positive linear transformation. SEU received much attention for several decades. The first complete axiomatic characterization of SEU was provided by Savage (1954), and many after him formulated preference conditions describing SEU (e.g. Anscombe \& Aumann, 1963; Wakker, 1984, 1989; d 'Aspremont \& Gevers, 1990; Gul, 1992).

Choquet expected utility (CEU) holds if the representing function has the following form:

$$
\left(f_{1}, \ldots, f_{n}\right) \mapsto i=1 \sum^{n} \pi_{\rho, i} \cdot U\left(f_{\rho(i)}\right)
$$

Here, outcomes are first rank-ordered and then they are valued by the representing function. $U$ is again cardinal. The $\pi_{\rho, j}$, for $j=1, \ldots, n$ are decision weights defined as follows

$$
\pi_{\rho, i}=\nu(\{\rho(1), \ldots, \rho(i)\})-\nu(\{\rho(1), \ldots, \rho(i-1)\}),
$$

where $\nu: 2^{S} \rightarrow[0,1]$ is a capacity, i.e. $\nu(\emptyset)=0, \nu(S)=1$ and $\nu(A \cup B) \geqslant \nu(A)$ for all events $A, B$. Under CEU the capacity is unique. Consequently, the decision weights are uniquely determined, they are nonnegative and sum to one for each permutation $\rho$. 
Capacities are nonadditive extensions of probability measures, thus, whenever $\nu$ in (1) is additive, CEU reduces to SEU above. CEU was introduced by Schmeidler (1989) for decision under uncertainty (see also Gilboa, 1987; Wakker, 1989; Nakamura, 1990, Chew \& Karni, 1994).

In this paper we focus on cumulative prospect theory (CPT). The representing CPTfunction is defined next. Let $f$ be an act such that for some $k \in\{0, \ldots, n\}$ and $\rho$ we have

$$
f_{\rho(1)} \geqslant \cdots \geqslant f_{\rho(k)} \geqslant 0>f_{\rho(k+1)} \geqslant \cdots \geqslant f_{\rho(n)},
$$

where $k=0$ means that all outcomes are negative and $k=n$ means that all outcomes are nonnegative. The CPT-function has the following form

$$
\begin{aligned}
\left(f_{1}, \ldots, f_{n}\right) \mapsto \quad & i=1 \sum^{k} \pi_{\rho, i}^{+} \cdot U\left(f_{\rho(i)}\right) \\
& +j=k+1 \sum^{n} \pi_{\rho, j}^{-} \cdot U\left(f_{\rho(j)}\right) .
\end{aligned}
$$

Here the continuous strictly increasing utility function $U$ is required to satisfy $U(0)=0$, and is a ratio scale, i.e. $U$ is unique up to multiplication by a positive real number. For the decision weights we have different uniquely determined capacities: $\nu^{+}$for gains and $\nu^{-}$for losses. They are defined as follows. For $i \leqslant k$ (the gain-part of $f$ ) we have similarly to CEU

$$
\pi_{\rho, i}^{+}=\nu^{+}(\{\rho(1), \ldots, \rho(i)\})-\nu^{+}(\{\rho(1), \ldots, \rho(i-1)\}) .
$$

For $j>k$ (the loss-part of $f$ ) we have

$$
\pi_{\rho, j}^{-}=\nu^{-}(\{\rho(j), \ldots, \rho(n)\})-\nu^{-}(\{\rho(j+1), \ldots, \rho(n)\}) .
$$

The CPT-value in (2) can be viewed as the sum of two CEU-values. The first sum is the CEU-value of the gain-part $f^{+}$with respect to the capacity $\nu^{+}$, and the second sum is the 
CEU-value of the loss-part $f^{-}$with respect to the dual of $\nu^{-}$(recall that here $\left.U(0)=0\right)$. The CEU-form for losses coincides with the original CEU-form, when in the definition of the decision weights $\nu^{-}(\{\rho(j), \ldots, \rho(n)\})$ is replaced by $1-\nu^{-}(S \backslash\{\rho(1), \ldots, \rho(j-1)\})$.

Moreover, we can write

$$
C P T(f)=C P T\left(f^{+}\right)+C P T\left(f^{-}\right)
$$

in agreement with Tversky \& Kahneman (1992). Aggregating those two values results in the final "worth" of the act. This feature is also exhibited in the proofs (see Appendix). First CPT will be established for gains and then CPT for losses is derived. Then, both parts merge into the general CPT-function.

In this section first preference axioms are formulated such that the utility function in (2) becomes an increasing "linear/exponential" function. A function $U: I R \rightarrow I R$ is from the increasing linear/exponential family for gains (losses) if one of the following holds for all $x \geqslant 0(x \leqslant 0)$ :

(i) $U(x)=\alpha \cdot x$, with $\alpha>0$,

(ii) $U(x)=\alpha \cdot e^{\lambda x}+\tau$, with $\alpha \cdot \lambda>0$ and $\tau \in \mathbb{R}$.

Under CPT utility satisfies $U(0)=0$. Therefore, in (i) we dropped the location parameter, and in (ii) the only possibility for the location parameter is $\tau=-\alpha$. In the above definition only the form of utility is described. Clearly the parameters $\alpha, \beta, \lambda$ can be different for gains than for losses.

The central property for a preference relation $\succcurlyeq$ to identify utility as a linear/exponential function is constant absolute risk aversion for gains and for losses. For gains (losses) it is defined as follows

$$
\left(f_{1}, \ldots, f_{n}\right) \succcurlyeq\left(g_{1}, \ldots, g_{n}\right) \Rightarrow\left(f_{1}+\varepsilon, \ldots, f_{n}+\varepsilon\right) \succcurlyeq\left(g_{1}+\varepsilon, \ldots, g_{n}+\varepsilon\right),
$$


whenever for all $i=1, \ldots, n$ the outcomes $f_{i}, f_{i}+\varepsilon, g_{i}, g_{i}+\varepsilon$ are gains (losses).

Before formulating the next preference condition some notation is introduced. For an event $I \subseteq S$ and $f, h \in \mathbb{R}^{n}$ by $h_{I} f$ we denote the act which results from $f$ by replacing $f_{i}$ with $h_{i}$ for each state $i \in I$; for states we write $h_{j} f$ instead of $h_{\{j\}} f$. We can now introduce the independence property for $\succcurlyeq$.

Definition The preference relation $\succcurlyeq$ on $\mathbb{I}^{n}$ satisfies tail independence if the following holds:

$$
a_{I} f \succcurlyeq a_{I} g \Leftrightarrow b_{I} f \succcurlyeq b_{I} g
$$

whenever $I=\{\rho(1), \ldots, \rho(m)\}$ or $I=\{\rho(l), \ldots, \rho(n)\}$ for some $m, l \in S$, and all acts in question are from the same set $\mathbb{R}_{\rho}^{n}$.

Tail independence requires that the preference between two acts is independent of common outcomes if, first, the acts are rank-ordered with respect to the same permutation, and second, if after rank-ordering those acts have their common outcomes placed in the first $m$ or last $(n-l+1)$ consecutive states. Thus, tail independence not only restricts the sure thing principle of Savage (1954) to comonotonic acts (called comonotonic independence in Chew \& Wakker, 1996), but in addition it further restricts comonotonic independence to hold for states in which common outcomes are best or worst. Cumulative prospect theory satisfies tail independence as is shown in the next lemma. In order to clarify the nature of CPT the proof is added into the main text.

LEMma 1 CPT implies tail independence.

Proof: Under CPT we have

$$
f \succcurlyeq g \Leftrightarrow C P T\left(f^{+}\right)+C P T\left(f^{-}\right) \geqslant C P T\left(g^{+}\right)+C P T\left(g^{-}\right),
$$


for all acts $f, g \in \mathbb{R}^{n}$.

Let $f, g \in \mathbb{R}_{\rho}^{n}$ for some permutation $\rho$ of the states. The following is implied by the above equivalence

$i=1 \sum^{k} \pi_{\rho, i}^{+} \cdot U\left(f_{\rho(i)}\right)+i=k+1 \sum^{n} \pi_{\rho, i}^{-} \cdot U\left(f_{\rho(i)}\right) \geqslant i=1 \sum^{k} \pi_{\rho, i}^{+} \cdot U\left(g_{\rho(i)}\right)+i=k+1 \sum^{n} \pi_{\rho, i}^{-} \cdot U\left(g_{\rho(i)}\right)$.

Assuming now that $f, g$ have common best outcomes, i.e. for some $m \in S$ we have $f_{\rho(i)}=g_{\rho(i)}$ for all $i=1, \ldots, m$, we conclude that the inequality is independent of the first $m$ summands. Hence, for $i \in I:=\{1, \ldots, m\}$, we can replace $f_{\rho(i)}, g_{\rho(i)}$ by arbitrary common outcomes $h_{\rho(i)}$ without affecting that inequality. Therefore, we can choose $h_{\rho(i)}$, such that $h_{\rho(I)} f, h_{\rho(I)} g \in \mathbb{R}_{\rho}^{n}$, implying

$$
f \succcurlyeq g \Leftrightarrow h_{\rho(I)} f \succcurlyeq h_{\rho(I)} g
$$

Because $\rho$ and $m$ are arbitrarily chosen, independence of common best outcomes holds. Similarly we can show that independence holds for common worst outcomes, which then implies tail independence of $\succcurlyeq$, and thus completes the proof.

The preference relation $\succcurlyeq$ on $\mathbb{R}^{n}$ satisfies monotonicity if $f \succ g$ whenever $f_{i} \geqslant g_{i}$ for all states $i$ with a strict inequality for at least one state.

The continuity condition defined here is with respect to the Euclidean topology on $\mathbb{R}^{n}$ : $\succcurlyeq$ satisfies continuity if for any act $f$ the sets $\left\{g \in \mathbb{R}^{n} \mid g \succcurlyeq f\right\}$ and $\left\{g \in \mathbb{R}^{n} \mid g \preccurlyeq f\right\}$ are closed subsets of $\mathbb{R}^{n}$.

TheOREm 2 Assume $n \geqslant 3$. For a preference relation $\succcurlyeq$ on $\mathbb{R}^{n}$ the following two statements are equivalent:

(i) CPT holds, with a continuous strictly increasing linear/exponential utility and positive decision weights. 
(ii) The preference relation satisfies the following conditions: weak ordering, monotonicity, continuity, tail independence, and constant absolute risk aversion for gains and for losses.

If (i) holds then the capacities are uniquely determined and the utility function is a ratio scale.

Next, we concentrate on the CPT-model with "power" utility. It is the most-used nonexpected utility form nowadays. For references see Wakker and Zank (1998). They provided an axiomatization of CPT with power utility for decision under risk. Here an extension of their results to decision under uncertainty is given.

A function $U: I R \rightarrow I R$ is from the positive power family for gains if

$$
U(x)=\alpha^{+} \cdot x^{\lambda^{+}}, \text {with } \alpha^{+}, \lambda^{+}>0, \text { forall } x \geqslant 0,
$$

and it is from the positive power family for losses if

$$
U(x)=-\alpha^{-} \cdot|x|^{\lambda^{-}}, \text {with } \alpha^{-}, \lambda^{-}>0, \text { forall } x \leqslant 0 .
$$

Recall that under CPT we require strict increasingness and $U(0)=0$ for the utility function. Hence, in the above definitions all parameters are positive and no location parameter is added.

The property of $\succcurlyeq$ which determines power utility is constant proportional risk aversion for gains and for losses. For gains (losses) it is defined as follows

$$
\left(f_{1}, \ldots, f_{n}\right) \succcurlyeq\left(g_{1}, \ldots, g_{n}\right) \Rightarrow\left(\varepsilon \cdot f_{1}, \ldots, \varepsilon \cdot f_{n}\right) \succcurlyeq\left(\varepsilon \cdot g_{1}, \ldots, \varepsilon \cdot g_{n}\right),
$$

for all $\varepsilon>0$ whenever all outcomes are gains (losses).

TheOREm 3 Assume $n \geqslant 3$. For a preference relation $\succcurlyeq$ on $\mathbb{I}^{n}$ the following two statements are equivalent: 
(i) CPT holds, with a positive power utility for gains and for losses, and positive decision weights.

(ii) The preference relation satisfies the following conditions: weak ordering, monotonicity, continuity, tail independence, and constant proportional risk aversion for gains and for losses.

If (i) holds then the capacities are uniquely determined, and the utility function is a ratio scale.

\section{CPT with Multilinear and Additive/Multiplicative Utility}

Recall that in this paper we consider a finite set of states, $S=\{1, \ldots, n\}$ for a natural number $n \geqslant 3$, where exactly one state is true and there is uncertainty about which is the true state. An act $f$ assigns to each state $j$ an outcome $f_{j}$. In this section we denote the set of outcomes by $X$, and $X$ is the product of a finite number of nondegenerate intervals $X_{1}, \ldots, X_{r}, r \geqslant 2$, called attribute sets. An outcome $x \in X$ can be written as a tuple $x=\left(x^{1}, \ldots, x^{r}\right)$ with attribute $x^{t} \in X_{t}$. Again we can view the set of acts as the product $X^{n}$. Sometimes we identify the constant act $(x, \ldots, x) \in X^{n}$ with the outcome $x \in X$.

For simplicity, we assume that each attribute $X_{t}$ contains the zero value in its interior. Therefore, $X$ contains the zero outcome, which is the status quo. Actually any other outcome in $X$ can play the role of the status quo. However, by rescaling the values in each attribute set we can ensure that the zero outcome becomes the status quo. Moreover, for money, zero as status quo is widely accepted in empirical work (see Kahneman \& Tversky, 1979). 
On the set of acts $X^{n}$ we assume a preference relation denoted by $\succcurlyeq$. The restriction of $\succcurlyeq$ to the constant acts (and therefore to $X$ ) is also denoted by $\succcurlyeq$. Gains and losses are now defined not only with respect to the status quo, but also depend on the preference relation $\succcurlyeq$ on $X$. Outcomes $x \succ 0$ are gains and outcomes $x \prec 0$ are losses. Note that here gains and losses can contain both positive and negative attributes. Therefore, the "aggregated worth" of an outcome among its $r$ attributes indicates if the outcome is a gain or a loss or is indifferent to the status quo.

Rank-ordering is also defined with respect to the preference relation $\succcurlyeq$ on $X$ : an act $f=\left(f_{1}, \ldots, f_{n}\right)$ is rank-ordered if $f_{1} \succcurlyeq \cdots \succcurlyeq f_{n}$. Similar to section 2 we denote by $X_{\rho}^{n}$ the set of acts that are rank-ordered according to $\rho$, where $\rho$ is a permutation of the states $\{1, \ldots, n\}$

Weak ordering, continuity and tail-independence are defined analogously to section 2 . The preference relation $\succcurlyeq$ on $X^{n}$ satisfies outcome-monotonicity if for all acts $f, g \in X^{n}$, $f_{i} \succcurlyeq g_{i}$ for all states $i$ implies $f \succcurlyeq g$, with a strict preference if for a state $j$ we have $f_{j} \succ g_{j}$. The preference relation $\succcurlyeq$ on $X^{n}$ satisfies attribute-monotonicity if for all outcomes $x, y \in X,\left[x \neq y\right.$ and $x^{t} \geqslant y^{t}$ for all $\left.t=1, \ldots, r\right]$ implies $x \succ y$.

For a subset $T$ of $\{1, \ldots, r\}$ and outcomes $z, x \in X$ we define $z^{T} x$ as the outcome with attribute $z^{t}$ for $t \in T$ and $x^{t}$ for $t \in T^{c}$, where $T^{c}:=\{1, \ldots, r\} \backslash T$. We denote by $X_{T}$ a factor, which is defined as $X_{T}:=\left\{X_{t} \mid t \in T\right\}$. Instead of $X_{\{t\}}$ we use $X_{t}$. In this section the central property is utility independence for factors restricted to rank-ordered sets, defined next:

Definition Let $T \subseteq\{1, \ldots, r\}$. The factor $X_{T}$ is utility independent for gains (losses) if

$$
\left(x^{T^{c}} f_{1}, \ldots, x^{T^{c}} f_{n}\right) \succcurlyeq\left(x^{T^{c}} g_{1}, \ldots, x^{T^{c}} g_{n}\right)
$$




$$
\begin{aligned}
& \Leftrightarrow \\
\left(y^{T^{c}} f_{1}, \ldots, y^{T^{c}} f_{n}\right) & \succcurlyeq\left(y^{T^{c}} g_{1}, \ldots, y^{T^{c}} g_{n}\right),
\end{aligned}
$$

holds, whenever all acts in question are contained in $X_{\rho}^{n}$ for some $\rho$, and all outcomes are gains (losses).

This property determines the following family of utility functions ( Theorem 4). A function $U: X \rightarrow \mathbb{R}$ is multilinear if there exist functions $U_{t}: X_{t} \rightarrow \mathbb{R}$ for $t=1, \ldots, r$ and constants $\delta_{T} \in \mathbb{R}$ for all $T \subseteq\{1, \ldots, r\}$ such that $U\left(x^{1}, \ldots, x^{r}\right)=\sum_{T \subseteq\{1, \ldots, r\}} \delta_{T}$. $\prod_{t \in T} U_{t}\left(x^{t}\right)$

Utility independence is a central tool in Keeney \& Raiffa's (1976) multiattribute utility theory. We define the property not only according to the separation into gains and losses but also restrict it to rank-ordered acts.

TheOREm 4 Assume $n \geqslant 3$. For a preference relation $\succcurlyeq$ on $X^{n}$ the following two statements are equivalent:

(i) CPT holds, with a continuous multilinear utility, strictly increasing in each attribute, and with positive decision weights.

(ii) The preference relation satisfies the following conditions: weak ordering, outcomemonotonicity, attribute-monotonicity, continuity, tail independence, and for each factor $X_{t}, t=1, \ldots, r$, utility independence for gains and for losses.

If (i) holds then the capacities are uniquely determined and the utility function is a ratio scale.

Mutual utility independence holds for gains (losses) whenever $X_{T}$ is utility independent for gains (losses) for all $T \subseteq\{1, \ldots, r\}$. This property characterizes additive/ multiplicative utilities (Theorem 5). A function $U: X \rightarrow I R$ is additive if $U\left(x^{1}, \ldots, x^{r}\right)=\sum_{t=1}^{r} U_{t}\left(x^{t}\right)$ 
and is multiplicative if $U\left(x^{1}, \ldots, x^{r}\right)=\prod_{t=1}^{r} U_{t}\left(x^{t}\right)$, where $U_{t}: X_{t} \rightarrow I R$ for all $t=1, \ldots, r$. A function is additive/multiplicative if it is either additive or multiplicative.

TheOREm 5 Assume $n \geqslant 3$. For a preference relation $\succcurlyeq$ on $X^{n}$ the following two statements are equivalent:

(i) CPT holds, with a continuous additive/multiplicative utility, strictly increasing in each attribute, and with positive decision weights.

(ii) The preference relation satisfies the following conditions: weak ordering, outcomemonotonicity, attribute-monotonicity, continuity, tail independence, and mutual utility independence for gains and for losses.

If (i) holds then the capacities are uniquely determined and the utility function is a ratio scale.

\section{Appendix}

Proof of Theorem 2: First, statement (i) is assumed, and statement (ii) is concluded: CPT holds for $\succcurlyeq$ on $\mathbb{R}^{n}$ with continuous increasing linear/exponential utility. Weak ordering is immediate from the existence of the representing CPT-function for $\succcurlyeq$. Monotonicity holds because utility is increasing and the decision weights are positive. Continuity of utility implies continuity of $\succcurlyeq$. Tail independence holds by Lemma 1. Finally, constant absolute risk aversion for gains and losses is implied by the nature of the utility function. This completes the proof of statement (ii).

Next, I assume statement (ii), and prove statement (i). The proof consists of several intermediate results. First, it is shown that on the set of rank-ordered acts $\mathbb{R}_{i d}^{n}$ the 
preference relation is represented by the additive function described in Lemma 6 . Then (Lemma 7), it is shown that the additive function in Lemma 6, when restricted to rankordered acts with nonnegative outcomes, agrees with the restriction of a CEU-function, where utility is linear/exponential and the decision weights are positive. Similarly, in Lemma 8 it is shown that the additive function of Lemma 6 , when restricted to rankordered acts with nonpositive outcomes, agrees with a CEU-restriction, where utility is linear/exponential and the decision weights are positive. In Lemma 7 similar results are derived for $\succcurlyeq$ on $\mathbb{R}_{\rho}^{n}$, for each permutation $\rho$ of the states. Then, it is shown that the different restrictions fit together into a general function, such that on $I R_{+}^{n}$ (the set of acts with nonnegative outcomes) and on $\mathbb{R}_{-}^{n}$ (the set of acts with nonpositive outcomes) Choquet expected utility holds, and thus on $\mathbb{R}^{n} \mathrm{CPT}$ holds for $\succcurlyeq$. We complete the proof of statement (i) by deriving uniqueness results .

LEMma 6 The preference relation $\succcurlyeq$ on $\mathbb{R}_{i d}^{n}$ is represented by the additive function

$$
\left(f_{1}, \ldots, f_{n}\right) \mapsto \sum_{j=1}^{n} V_{j}\left(f_{j}\right)
$$

with continuous strictly increasing functions $V_{1}, \ldots, V_{n}: I R \rightarrow I R$, which are uniquely determined satisfying $V_{j}(0)=0$ for all $j$ and $\sum_{j=1}^{n} V_{j}(1)=1$.

Proof: The proof follows immediately from Lemma 7 in Wakker \& Zank (1997). There, the statement is formulated for a preference relation on a set of simple lotteries (i.e. finite probability distributions over $I R$ ) with rank-ordered outcomes. However, they fix a finite probability distribution, such that only outcomes can vary, which results in a set isomorphic to $\mathbb{R}_{i d}^{n}$. Then our statement results.

LEMMA 7 On the set of rank-ordered acts with nonnegative outcomes the representation 
of Lemma 6 agrees with the following function

$$
C E U_{i d}^{+}:\left(f_{1}, \ldots, f_{n}\right) \mapsto j=1 \sum^{n} \pi_{i d, j}^{+} \cdot U_{i d}\left(f_{j}\right),
$$

where $U_{i d}$ is a strictly increasing linear/exponential utility function, satisfying $U_{i d}(0)=0$ and $U_{i d}(1)=1$, and the decision weights $\pi_{i d, j}^{+}$, for $j=1, \ldots, n$, are all positive. Utility and the decision weights are uniquely determined.

Proof: We have given the preference relation $\succcurlyeq$ on $\mathbb{R}_{i d}^{n}$, which is represented by the function $\sum_{j=1}^{n} V_{j}$ with the $V_{j}$ 's as described in Lemma 6 . Moreover $\succcurlyeq$ satisfies constant absolute risk aversion on $I R_{++, i d}^{n}$, i.e. the set of rank-ordered acts with positive outcomes.

We define $U_{i d}(x)=\sum_{j=1}^{n} V_{j}(x)$ for all nonnegative $x$. Therefore, by Lemma $6 U_{i d}$ becomes unique satisfying $U_{i d}(0)=0, U_{i d}(1)=1$.

Let us fix some $1 \leqslant i<n$. We restrict our analysis to acts with identical outcomes for the first $i$ states and for the last $n-i$ states, i.e. to acts $f$ with $f_{j}=x$ for $j=1, \ldots, i$ and $f_{j}=y$ for $j=i+1, \ldots, n$, for outcomes $x, y$ with $x \geqslant y \geqslant 0$. We denote these acts by $(x, y)_{i}$. On this two-dimensional subset the preference relation is represented by $(x, y)_{i} \mapsto Z_{i}(x)+W_{i}(y)$, with $Z_{i}:=\sum_{j=1}^{i} V_{j}$ and $W_{i}:=\sum_{j=i+1}^{n} V_{j}$. Constant absolute risk aversion for gains implies that $\succcurlyeq$ on this subset is invariant w.r.t. addition of a common constant to all outcomes (whenever the resulting acts remain in this subset). Considering only gains $(y>0)$, Miyamoto \& Wakker (1996, Theorem 1$)$ show that $Z_{i}$ and $W_{i}$ are proportional, which obviously remains valid when the zero outcome is included. Moreover, $Z_{i}$ and $W_{i}$ are proportional to their sum, which is $U_{i d}$. Therefore, by Miyamoto \& Wakker, they are of the form $Z_{i}=\pi_{Z_{i}} \cdot U_{i d}$ and $W_{i}=\pi_{W_{i}} \cdot U_{i d}$, for positive uniquely determined $\pi_{Z_{i}}, \pi_{W_{i}}$, which sum to one. Further, Miyamoto \& Wakker concluded that the utility function $U_{i d}$ is from the increasing linear/exponential family for gains. This analysis holds for any fixed $1 \leqslant i<n$. 
We define now $\pi_{i d, 1}^{+}:=\pi_{Z_{1}}, \pi_{i d, j}^{+}:=\pi_{Z_{j}}-\pi_{Z_{j-1}}$ for $j=2, \ldots, n-1$, and $\pi_{i d, n}^{+}:=\pi_{W_{n-1}}$. Monotonicity implies that all the $\pi_{i d, j}^{+}$'s are positive. By their definition they are uniquely determined and sum to one. Then, we can compute $V_{1}=Z_{1}=\pi_{i d, 1}^{+} \cdot U_{i d}$, and inductively $V_{j}=Z_{j}-Z_{j-1}=\pi_{i d, j}^{+} \cdot U_{i d}$ for $j=2, \ldots, n-1$, and $V_{n}=W_{n-1}=\pi_{i d, n}^{+} \cdot U_{i d}$

Finally, from this analysis, we conclude that on $\mathbb{R}_{+, i d}^{n}$ the representation of Lemma 6 agrees with the function in (3). This concludes the proof of Lemma 7.

LEMMA 8 On the set of rank-ordered acts with nonpositive outcomes the representation of Lemma 6 agrees with the following function

$$
C E U_{i d}^{-}:\left(f_{1}, \ldots, f_{n}\right) \mapsto j=1 \sum^{n} \pi_{i d, j}^{-} \cdot U_{i d}\left(f_{j}\right),
$$

where $U_{i d}$ is a strictly increasing linear/exponential utility function, satisfying $U_{i d}(0)=0$, and the decision weights $\pi_{i d, j}^{-}$, for $j=1, \ldots, n$, are all positive. Utility and the decision weights are uniquely determined.

Proof: The proof is similar to the one of Lemma 7. We can view the "problem" $\left\{S, R_{-, i d}^{n}, \succcurlyeq, \sum_{j=1}^{n} V_{j}(\cdot)\right\}$ as an equivalent problem $\left\{S^{*}, \mathbb{R}_{+, i d}^{n}, \succcurlyeq^{*},-\sum_{j=n}^{1} V_{j}(-(\cdot))\right\}$. Now $S^{*}:=\{n, \ldots, 1\}$, an act $\left(f_{1}, \ldots, f_{n}\right) \in \mathbb{R}_{-, i d}^{n}$ corresponds to an act $\left(-f_{n}, \ldots,-f_{1}\right) \in \mathbb{R}_{+, i d}^{n}$ and the preference relation $\succcurlyeq^{*}$ is defined as follows:

$$
\left(-f_{n}, \ldots,-f_{1}\right) \succcurlyeq^{*}\left(-g_{n}, \ldots,-g_{1}\right) \Leftrightarrow\left(f_{1}, \ldots, f_{n}\right) \preccurlyeq\left(g_{1}, \ldots, g_{n}\right) .
$$

Therefore $\succcurlyeq^{*}$ is a continuous monotonic weak order satisfying tail independence and constant absolute risk aversion for gains. Moreover, $\succcurlyeq^{*}$ is represented by

$$
\left(-f_{n}, \ldots,-f_{1}\right) \mapsto-\sum_{j=n}^{1} V_{j}\left(f_{j}\right)
$$

with the $V_{j}$ 's as described in Lemma 6 . It can now be demonstrated, following the lines in the proof of Lemma 7, that similar results as in Lemma 7 hold here. Reformulation in 
terms of the problem $\left\{S, \mathbb{R}_{-, i d}^{n}, \succcurlyeq, \sum_{j=1}^{n} V_{j}(\cdot)\right\}$ gives exactly the statement of Lemma 8 . This completes the proof.

Lemma 9 For each permutation $\rho$ of $\{1, \ldots, n\}$ the preference relation $\succcurlyeq$ on $\mathbb{I}_{\rho}^{n}$ is represented by an additive function

$$
\left(f_{1}, \ldots, f_{n}\right) \mapsto j=1 \sum^{n} V_{j}^{\rho}\left(f_{\rho(j)}\right),
$$

with continuous strictly increasing functions $V_{1}^{\rho}, \ldots, V_{n}^{\rho}: \mathbb{R} \rightarrow \mathbb{R}$, which are uniquely determined satisfying $V_{j}^{\rho}(0)=0$ for all $j$ and $\sum_{j=1}^{n} V_{j}^{\rho}(1)=1$. The additive function described above agrees on $\mathbb{R}_{+, \rho}^{n}$ with the following function

$$
C E U_{\rho}^{+}:\left(f_{1}, \ldots, f_{n}\right) \mapsto j=1 \sum^{n} \pi_{\rho, j}^{+} \cdot U_{\rho}\left(f_{\rho(j)}\right)
$$

and on $\mathbb{R}_{-, \rho}^{n}$ with the function

$$
C E U_{\rho}^{-}:\left(f_{1}, \ldots, f_{n}\right) \mapsto j=1 \sum^{n} \pi_{\rho, j}^{-} \cdot U_{\rho}\left(f_{\rho(j)}\right)
$$

The utility function $U_{\rho}$ is from the increasing linear/exponential family for both gains and losses, satisfying $U_{\rho}(0)=0$ and $U_{\rho}(1)=1$, and the decision weights $\pi_{\rho, j}^{+}, \pi_{\rho, j}^{-}$, for $j=1, \ldots, n$, are all positive. Utility and the decision weights are uniquely determined.

Proof: Take any permutation $\rho$ of $\{1, \ldots, n\}$. The preference relation $\succcurlyeq$ on $\mathbb{R}_{\rho}^{n}$ is a continuous monotonic weak order, satisfying tail independence and constant absolute risk aversion for gains and for losses. The proofs of Lemma 6, 7 and 8 can be adapted, considering instead of $S$ the "reordered" set of states $S_{\rho}:=\left\{\rho^{-1}(1), \ldots, \rho^{-1}(n)\right\}$.

We have now obtained representations for $\succcurlyeq$ on each set $I R_{\rho}^{n}$, which, on $\mathbb{R}_{+, \rho}^{n}$ and $\mathbb{R}_{-, \rho}^{n}$ agree with restrictions of CEU-functions as described in Lemma 9. The next step in the proof of Theorem 2 is to show that the different functions have identical utility. We show this only for gains. A similar result can be proved for losses. 
Let $\rho$ be a permutation of the states $\{1, \ldots, n\}$, such that the set $\mathbb{R}_{+, \rho}^{n} \cap \mathbb{R}_{+, i d}^{n}$ contains nonconstant acts. Then, $C E U_{\rho}^{+}$and $C E U_{i d}^{+}$jointly represent the preference relation $\succcurlyeq$ on the intersection $\mathbb{R}_{+, \rho}^{n} \cap \mathbb{R}_{+, i d}^{n}$. However, any additive representation of $\succcurlyeq$ on $\mathbb{R}_{+, \rho}^{n} \cap$ $I R_{+, i d}^{n}$, because cardinal (see for instance Chateauneuf \& Wakker, 1993), becomes unique by fixing scale and location. Moreover, by reasonings similar to Lemma 9, uniquely determined utility and decision weights can be derived for such a representation. Thus, the representation for $\succcurlyeq$ on $\mathbb{R}_{+, \rho}^{n} \cap \mathbb{R}_{+, i d}^{n}$, with unique utility and decision weights, is a restriction of both the representation for $\succcurlyeq$ on $\mathbb{R}_{+, \rho}^{n}$ and the representation for $\succcurlyeq$ on $\mathbb{R}_{+, i d}^{n}$. Consequently, the utilities for both extensions are identical and we conclude that $U_{\rho}=U_{i d}$ on $\mathbb{R}$, whenever $\mathbb{R}_{+, \rho}^{n} \cap \mathbb{R}_{+, i d}^{n}$ contains nonconstant acts.

Now let $\rho$ be a permutation of $\{1, \ldots, n\}$, such that the set $I R_{+, \rho}^{n} \cap I R_{+, i d}^{n}$ contains only constant acts. Then, using the fact that $n \geqslant 3$, one can easily construct a sequence of permutations $\rho_{1}, \rho_{2}, \rho_{3}$ of the states such that all sets $I R_{+, \rho}^{n} \cap \mathbb{R}_{+, \rho_{1}}^{n}, \mathbb{I} R_{+, \rho_{1}}^{n} \cap \mathbb{R}_{+, \rho_{2}}^{n}$, $\mathbb{R}_{+, \rho_{2}}^{n} \cap \mathbb{R}_{+, \rho_{3}}^{n}, \mathbb{R}_{+, \rho_{3}}^{n} \cap \mathbb{R}_{+, i d}^{n}$ contain nonconstant acts. Hence, by the analysis before, we conclude that the utilities for $C E U_{\rho}^{+}$and $C E U_{i d}^{+}$are identical.

Therefore, we conclude that the different functions derived in Lemma 9, have the same utility function $U$, and hence assign the same value to constant acts, i.e. the acts which are commonly contained in all sets $\mathbb{R}_{\rho}^{n}$. Using this result, the following holds:

LEMma 10 For each act $f \in \mathbb{R}_{+}^{n}$ or $\mathbb{R}_{-}^{n}$ there exists a certainty equivalent, i.e. a constant act $x_{f}$ with $f \sim x_{f}$.

Proof (only for the case $f \in \mathbb{R}_{+}^{n}$ ): Take any act $f \in \mathbb{R}_{+, \rho}^{n}$. Let $x$ be the maximal and $y$ the minimal outcome of $f$. Monotonicity of $\succcurlyeq$ implies $x \succcurlyeq f \succcurlyeq y$. Thus, the following equivalence holds

$$
C E U_{\rho}^{+}(x) \geqslant C E U_{\rho}^{+}(f) \geqslant C E U_{\rho}^{+}(y) \Leftrightarrow U(x) \geqslant C E U_{\rho}^{+}(f) \geqslant U(y),
$$


and, by continuity of $U$, there exists $x_{f} \in \mathbb{R}_{+}$, with $C E U_{\rho}^{+}(f)=U\left(x_{f}\right)$. By monotonicity, $x_{f}$ is unique. Hence, the constant act $x_{f}$ is indifferent to $f$.

Now we show the existence of a representing CEU-function for $\succcurlyeq$ on $I R_{+}^{n}$. Take any acts $f, g \in \mathbb{R}_{+}^{n}$. Let be $f \in \mathbb{R}_{+, \rho}^{n}, g \in \mathbb{R}_{+, \rho^{\prime}}^{n}$ and $C E U_{\rho}^{+}, C E U_{\rho^{\prime}}^{+}$the representations for $\succcurlyeq$ on $I R_{+, \rho}^{n}, \mathbb{R}_{+, \rho^{\prime}}^{n}$, respectively, derived in Lemma 9. Then, by Lemma 10 we have $f \succcurlyeq g \Leftrightarrow$ $f \succcurlyeq x_{g} \Leftrightarrow C E U_{\rho}^{+}(f) \geqslant C E U_{\rho}^{+}\left(x_{g}\right)$. Further, because utility is the same for all CEU-forms, it follows that $C E U_{\rho}^{+}\left(x_{g}\right)=U\left(x_{g}\right)=C E U_{\rho^{\prime}}^{+}\left(x_{g}\right)=C E U_{\rho^{\prime}}^{+}(g)$. Thus, we conclude

$$
f \succcurlyeq g \Leftrightarrow C E U_{\rho}^{+}(f) \geqslant C E U_{\rho^{\prime}}^{+}(g) \text {. }
$$

By setting $f=g$, this implies that $C E U_{\rho}^{+}$agrees with $C E U_{\rho^{\prime}}^{+}$on common domain, i.e. on $\mathbb{R}_{+, \rho}^{n} \cap \mathbb{R}_{+, \rho^{\prime}}^{n}$. Thus, they can be considered restrictions of one function. Because $f$ and $g$ were arbitrary, we conclude the existence of a general function, denoted $C E U^{+}$, which represents $\succcurlyeq$ on $\mathbb{R}_{+}^{n}$, and agrees with $C E U_{\rho}^{+}$on $\mathbb{R}_{+, \rho}^{n}$ for any permutation $\rho$ of the states.

Recall that the act $1_{I} 0$ assigns outcome 1 to the states in $I$ and outcome 0 elsewhere. Now define $\nu^{+}(I):=C E U^{+}\left(1_{I} 0\right)$ for all $I \subseteq\{1, \ldots, n\}$. Obviously, $\nu^{+}$is a capacity on $S$. Moreover the following holds

$$
\pi_{\rho, j}^{+}=\nu^{+}(\rho(1), \ldots, \rho(j))-\nu^{+}(\rho(1), \ldots, \rho(j-1))
$$

for $j=1, \ldots, n$ and any permutations $\rho$ of the states. Because the decision weights are uniquely determined, by the above definition the capacity $\nu^{+}$is unique.

Let us summarize: The preference relation $\succcurlyeq$ on $I R_{+}^{n}$ is represented by the function $C E U^{+}$which is a Choquet expected utility function as described in (1). Further the utility function $U$ is uniquely determined from the increasing linear/exponential family for gains, satisfying $U(0)=0, U(1)=1$, and the capacity $\nu^{+}$on $S$ generates positive decision weights. 
Similarly, for the preference relation $\succcurlyeq$ on $\mathbb{R}_{-}^{n}$, we can derive representability by a Choquet expected utility function, now denoted $C E U^{-}$. The utility function $U$ is from the increasing linear/exponential for losses, it is again unique and satisfies $U(0)=0$. The capacity $\nu^{-}$, now defined by $\nu^{-}(I):=C E U^{-}\left(-1_{I} 0\right) / U(-1)$ for all $I \subseteq\{1, \ldots, n\}$ is again unique, and it generates positive decision weights. Moreover the following holds for $\nu^{-}$

$$
\pi_{\rho, j}^{-}=\nu^{-}(\rho(j), \ldots, \rho(n))-\nu^{-}(\rho(j+1), \ldots, \rho(n))
$$

for $j=1, \ldots, n$ and any permutations $\rho$ of the states.

Now let $f$ be an act containing both gains and losses. Suppose $f \in R_{\rho}^{n}$ for a permutation $\rho$ of $\{1, \ldots, n\}$. There exists a $k \in\{1, \ldots, n\}$ with

$$
f_{\rho(1)} \geqslant \cdots \geqslant f_{\rho(k)} \geqslant 0>f_{\rho(k+1)} \geqslant \cdots \geqslant f_{\rho(n)} .
$$

Then, with $f^{+}$the gain part of $f, f^{-}$the loss part of $f$, and the $V_{j}^{\rho}$ 's from Lemma 9 , the following holds

$$
j=1 \sum^{n} V_{j}^{\rho}\left(f_{\rho(j)}\right)=j=1 \sum^{n} V_{j}^{\rho}\left(f_{\rho(j)}^{+}\right)+j=1 \sum^{n} V_{j}^{\rho}\left(f_{\rho(j)}^{-}\right)
$$

which, by the results above, is equivalent to

$$
j=1 \sum^{n} V_{j}^{\rho}\left(f_{\rho(j)}\right)=C E U^{+}\left(f^{+}\right)+C E U^{-}\left(f^{-}\right) .
$$

Therefore, the additive representations for $\succcurlyeq$ on $R_{\rho}^{n}$ described in Lemma 9 can be considered as restrictions of a common function, defined by $f \mapsto C E U^{+}\left(f^{+}\right)+C E U^{-}\left(f^{-}\right)$. Obviously, this function represents the preference relation $\succcurlyeq$ on the entire set of acts $\mathbb{R}^{n}$, and it is a CPT-function as described in (2), with a increasing linear/exponential utility function for gains and for losses $U$, which satisfies $U(0)=0$ and $U(1)=1$. Utility and the capacities $\nu^{+}, \nu^{-}$are uniquely determined by the analysis made separately for gains and for losses. 
Note that in the derivation of the representation above we have always fixed scale and location. Let now $U^{*}, \mu^{+}, \mu^{-}$describe a CPT-function representing $\succcurlyeq$ on $\mathbb{R}^{n}$, with $U^{*}(0)=0$. Thus, only location is fixed for $U^{*}$ but not scale. Then, also $U^{*} / U^{*}(1), \mu^{+}, \mu^{-}$ describes a CPT-function representing $\succcurlyeq$ on $\mathbb{R}^{n}$. Consequently, by the results before, $U^{*} / U^{*}(1)=U$, and $\mu^{+}=\nu^{+}, \mu^{-}=\nu^{-}$follows. Further, if $U, \nu^{+}, \nu^{-}$describe a CPTrepresentation for $\succcurlyeq$ on $\mathbb{R}^{n}$, then also $\gamma \cdot U, \nu^{+}, \nu^{-}$describe a CPT-representation for $\succcurlyeq$ on $\mathbb{R}^{n}$ for any positive $\gamma$. This shows that $U$ is a ratio scale, and that the capacities are uniquely determined. Moreover all generated decision weights are positive. Thus the proof of statement (ii) is complete.

Hence, we conclude the proof of Theorem 2.

Proof of Theorem 3: That statement (i) implies (ii) is immediate. The proof of (i) from (ii) is analogous to that in the proof of Theorem 2. The difference is that for the preference relation here constant absolute risk aversion is replaced by constant proportional risk aversion. In Lemma 6 this is not yet relevant, therefore Lemma 6 holds here. Constant absolute risk aversion was relevant in Lemma 7. Considering constant proportional risk aversion instead, Lemma 7 remains valid if we replace " $U_{i d}$ is a strictly increasing linear/exponential utility function" by " $U_{i d}$ is from the positive power family". Then in the proof we have to use Theorem 2 of Miyamoto \& Wakker (1996) instead of their Theorem 1. Other than that, the proof can entirely be adapted with a positive power utility $U_{i d}$ instead of a linear exponential one.

Similarly, the Lemmas 8 and 9 remain valid with power utility instead of linear/exponential utility. Moreover, the analysis following the proof of Lemma 9 can entirely be repeated here, concluding statement (i) in Theorem 3. This completes the proof.

Proof of Theorem 4: I assume statement (i), and conclude statement (ii). Suppose 
that CPT holds for $\succcurlyeq$ on $X^{n}$, with a continuous multilinear utility, strictly increasing in each attribute, and with positive decision weights. Weak ordering of $\succcurlyeq$ is immediate from the existence of the representing CPT-function for $\succcurlyeq$. Outcome-monotonicity holds the decision weights are positive. Attribute-monotonicity follows because utility is strictly increasing in each attribute, and because the decision weights are positive. Continuity of utility implies continuity of $\succcurlyeq$. Tail independence is given by Lemma 1 . Finally, on each set $X_{\rho}^{n}$ utility independence for each factor $X_{t}, t=1, \ldots, r$, for gains and losses is implied by the nature of the utility function. This completes the proof of statement (ii).

Now I prove that statement (ii) implies statement (i). The proof hereof mainly follows the lines indicated in the proof of statement (i) from (ii) of Theorem 2. I point out differences here, and whenever possible I refer to that proof.

The first difference consists in the existence of "extreme acts". An outcome $x \in X$ is maximal if for no other outcome $y \in X$, we have $y \succ x$, and $x$ is minimal if for no other outcome $y \in X$, we have $y \prec x$. An act assigning to each state a maximal outcome or to each state a minimal outcome is an extreme act. Wakker (1993) pointed out difficulties for additive representability on rank-ordered sets, in the presence of extreme acts. They had to be excluded in order to derive additive representability. Under proportionality of the functions in the additive representation on the set of nonminimal and nonmaximal outcomes, as will be derived here, extensions to extreme acts were possible (see Proposition 3.5 in Wakker, 1993).

LEMmA 11 The preference relation $\succcurlyeq$ on $X_{i d}^{n} \backslash\{$ extreme acts $\}$ is represented by the additive function

$$
\left(f_{1}, \ldots, f_{n}\right) \mapsto \sum_{j=1}^{n} V_{j}\left(f_{j}\right),
$$


with continuous functions $V_{1}, \ldots, V_{n}: X \rightarrow \mathbb{R}$, which preserve the ordering of outcomes, and are uniquely determined satisfying $V_{j}(0)=0$ for all $j$ and $\sum_{j=1}^{n} V_{j}(w)=1$ for some fixed $w \succ 0$.

Proof: The proof follows by similar reasonings to those used in the proof of Lemma 7 of Wakker \& Zank (1997). There it is described how tail independence implies, first locally then globally, the additivity axioms required in Chateauneuf \& Wakker (1993). Then by Corollary C.5 of Chateauneuf \& Wakker additive representability follows.

For $z \in X$ and $t=1, \ldots, r$ we define the sets

$$
X_{t,+}(z):=\left\{x^{t} \in X_{t} \mid x^{t} \geqslant z^{t}\right\} \text { and } X_{t,-}(z):=\left\{x^{t} \in X_{t} \mid x^{t} \leqslant z^{t}\right\} .
$$

Now take $z \in \operatorname{int}(X)$, i.e. the interior of $X$, with $z \sim 0$. We restrict our analysis to rank-ordered acts from $X_{+}^{n}(z) \backslash\{$ extreme acts $\}$ where $X_{+}(z):=\left[X_{1,+}(z)\right] \times \cdots \times\left[X_{r,+}(z)\right]$. Note that by attribute monotonicity the outcomes in $X_{+}(z)$ are all gains except for $z$, which by its choice is indifferent to the status quo.

LEMMA 12 On the set $X_{+, i d}^{n}(z) \backslash\{$ extreme acts $\}$ the representation of Lemma 11 agrees with the following function

$$
C E U_{i d, z}^{+}:\left(f_{1}, \ldots, f_{n}\right) \mapsto j=1 \sum^{n} \pi_{i d, j, z}^{+} \cdot U_{i d}^{z}\left(f_{j}\right),
$$

where $U_{i d}^{z}$ is a multilinear utility function, preserving the ordering on $X$, and increasing in each attribute. $U_{i d}^{z}$ satisfies $U_{i d}^{z}(z)=0$, and the decision weights $\pi_{i d, j, z}^{+}$for $j=1, \ldots, n$ are all positive. Utility and the decision weights are uniquely determined.

Proof: We have given the preference relation $\succcurlyeq$ on $X_{+, i d}^{n}(z) \backslash\{$ extreme acts $\}$, which is represented by the function $\sum_{j=1}^{n} V_{j}$ with the $V_{j}$ 's as described in Lemma 11. Moreover 
$\succcurlyeq$ satisfies attribute monotonicity and for each attribute set $X_{j,+}(z)$ utility independence for gains on $X_{+, i d}^{n}(z) \backslash\{$ extreme acts $\}$.

We define $U_{i d}^{z}(x)=\sum_{j=1}^{n} V_{j}(x)$ for all $x \in X_{+}(z)$. Therefore, by Lemma 11, $U_{i d}^{z}$ becomes unique and satisfies $U_{i d}^{z}(z)=0$.

Now the reasonings are similar to those in the proof of Lemma 7 . Instead of $x \geqslant y \geqslant 0$ we have $x \succcurlyeq y \succcurlyeq z$, instead of Theorem 1 of Miyamoto \& Wakker (1996) we use Theorem 5, and monotonicity is replaced by outcome-monotonicity. Except for these changes the proof of Lemma 7 can entirely be repeated here.

We conclude that on $X_{+, i d}^{n}(z) \backslash\{$ extreme acts $\}$ the representation of Lemma 11 agrees with the function in (7). This concludes the proof of Lemma 12.

Let now $\hat{z}, \tilde{z} \in \operatorname{int}(X)$ with $\hat{z} \sim \tilde{z} \sim 0$ be any distinct outcomes. (Such outcomes exist because $r \geqslant 2$, and because on $X$ the preference relation $\succcurlyeq$ is a continuous weak order, satisfying attribute-monotonicity.) Then, the outcome $y$, defined by $y^{t}:=\max \left\{\hat{z}^{t}, \tilde{z}^{t}\right\}$ for all $t=1, \ldots, r$, is contained in $\operatorname{int}(X)$, and by attribute monotonicity satisfies $y \succ 0$.

A similar analysis as in the proof of Lemma 12 can be made here, such that we can conclude that on $X_{+, i d}^{n}(y) \backslash\{$ extreme acts $\}$ the additive function in Lemma 11 agrees with

$$
C E U_{i d, y}^{+}:\left(f_{1}, \ldots, f_{n}\right) \mapsto j=1 \sum^{n} \pi_{i d, j, y}^{+} \cdot U_{i d}^{y}\left(f_{j}\right)
$$

with uniquely determined multilinear utility function $U_{i d}^{y}$ increasing in each attribute, and positive decision weights $\pi_{i d, j, y}^{+}$for $j=1, \ldots, n$.

Now, both $X_{+, i d}^{n}(\hat{z})$ and $X_{+, i d}^{n}(\tilde{z})$ contain $X_{+, i d}^{n}(y)$, and the latter contains an open subset where $C E U_{i d, \hat{z}}^{+}$and $C E U_{i d, \tilde{z}}^{+}$both represent the same preference. By the uniqueness of the function in (8), we conclude that the utilities and the decision weights in $C E U_{i d, \hat{z}}^{+}$ and $C E U_{i d, \tilde{z}}^{+}$are identical, i.e. $U_{i d}^{\hat{z}}=U_{i d}^{\tilde{z}}$ and $\pi_{i d, j, \hat{z}}^{+}=\pi_{i d, j, \tilde{z}}^{+}$for all $j=1, \ldots, n$. Moreover, 
because $\hat{z}$ and $\tilde{z}$ were arbitrarily chosen from $\operatorname{int}(X)$, we conclude that the function in $(7)$ is independent of $z$, and thus we can suppress the index $z$ in $(7)$.

Recall, that we restricted the above analysis to $z \in \operatorname{int}(X)$ such that $z \sim 0$. Doing so, the outcomes where $U_{i d}$ is not yet defined are boundary outcomes of $X$. But for these boundary outcomes, viewed as constant acts, the function $\sum_{j=1}^{n} V_{j}$ is defined (except for the extreme acts), and thus, we can continuously extend $U_{i d}$ to those outcomes by $U_{i d}:=\sum_{j=1}^{n} V_{j}$, preserving multilinearity

In Lemma 11 we excluded the extreme acts. However, later in the analysis, we concluded that the $V_{j}$ 's in Lemma 11 are proportional. Then, by Proposition 3.5 of Wakker (1993), we can extend $\sum_{j=1}^{n} V_{j}$ to the extreme acts which are gains, and thus, $U_{i d}$ to the entire set $X_{+, i d}$. Finally we conclude the following:

LEMma 13 On the set $X_{+, i d}^{n}$ the preference relation $\succcurlyeq$ is represented by the following function

$$
C E U_{i d}^{+}:\left(f_{1}, \ldots, f_{n}\right) \mapsto j=1 \sum^{n} \pi_{i d, j}^{+} \cdot U_{i d}\left(f_{j}\right),
$$

where $U_{i d}$ is a multilinear utility function, preserving the ordering on $X$, and increasing in each attribute; $U_{i d}$ satisfies $U_{i d}(0)=0, U_{i d}(w)=1$; the decision weights $\pi_{i d, j}^{+}$are positive for all $j=1, \ldots, n$. Utility and the decision weights are uniquely determined.

Now the rest of the proof of statement (i) follows the line of the proof of statement (i) in Theorem 2. The reasonings are similar, and therefore, we briefly indicate the next steps.

First, for each permutation $\rho$ of the states, on the set $X_{+, \rho}^{n}\left(X_{-, \rho}^{n}\right)$ we can derive representability of $\succcurlyeq$ by a function $C E U_{\rho}^{+}\left(C E U_{\rho}^{-}\right)$similar to the one in (9) where utility is fixed 0 at $0 \in X$ and 1 at $w \in X$. Secondly, we can show that the functions $C E U_{\rho}^{+}\left(C E U_{\rho}^{-}\right)$ are restrictions of a general CEU-function $C E U^{+}\left(C E U^{-}\right)$, with unique multilinear utility 
and unique capacity, representing $\succcurlyeq$ on $X_{+}^{n}\left(X_{-}^{n}\right)$. Third, we can derive CPT for $\succcurlyeq$ on $X^{n}$. Finally, we can prove the uniqueness results.

This completes the proof of Theorem 4.

Proof of Theorem 5: That statement (i) implies (ii) is immediate. The proof of (i) from (ii) is analogous to that in the proof of Theorem 4. The difference is that for the preference relation utility independence for attribute sets is replaced by mutual utility independence. In Lemma 11 this is not yet relevant, therefore Lemma 11 holds here. Utility independence for attribute sets was relevant in Lemma 12. Considering mutual utility independence instead, Lemma 12 remains valid if we replace " $U_{i d}^{z}$ is a multilinear utility function" by " $U_{i d}^{z}$ is additive/multiplicative utility function". Then in the proof we have to use Theorem 4 of Miyamoto \& Wakker (1996) instead of their Theorem 5. Other than that, the proof can entirely be adapted with a additive/multiplicative utility $U_{i d}^{z}$ instead of a multilinear one.

Similarly, Lemma 13 remains valid with additive/multiplicative utility instead of multilinear utility. Further, the analysis following the proof of Lemma 13 can entirely be repeated here, concluding statement (i) in Theorem 5. This completes the proof.

\section{References}

Anscombe, F.J.\& R.J. Aumann (1963), "A Definition of Subjective Probability", Annals of Mathematical Statistics 34 199-205.

Beetsma, R.M.W.J. \& P.C. Schotman (1998), "Measuring Risk Attitudes in a Natural Experiment: Data from the Television Game Show LINGO," Working Paper 98-49, Maastricht University, The Netherlands. 
Bell, D.E. (1988), "One-Switch Utility Functions and a Measure of Risk," Management Sciences 341416-1424.

Chateauneuf, A. \& P.P. Wakker (1993), "From Local to Global Additive Representation," Journal of Mathematical Economics 22 523-545.

Chew, S.H. \& E. Karni (1994), "Choquet Expected Utility with a Finite State Space: Commutativity and Act-Independence," Journal of Economic Theory 62 469-479.

Chew, S.H. \& P.P. Wakker (1996), "The Commonotonic Sure-Thing Principle," Journal of Risk and Uncertainty 12 5-27.

Currim, I.S. \& R.K. Sarin (1989), "Prospect Versus Utility," Management Science 35 $22-41$.

d'Aspremont, C.\& L. Gevers (1990), "Invariance, Neutrality and Weakly Continuous Expected Utility," in: J.J. Gabszewicz, J.-F. Richard and L.A. Wolsey, Chapter 4, 6788, Economic Decision Making: Games, Econometrics and Optimization, Elsevier Science Publishers B.V.

Farquhar, P.H. \& Y. Nakamura (1987), "Constant Exchange Risk Properties," Operations Research 35 206-214.

Gilboa, I. (1987) "Expected Utility with Purely Subjective Non-additive Probabilities," Journal of Mathematical Economics 16 65-88.

Gul, F. (1992) "Savage's Theorem with a Finite Number of States," Journal of Economic Theory 57 99-110.

Kahneman, D. \& A. Tversky (1979), "Prospect Theory: An Analysis of Decision under Risk," Econometrica 47 263-291.

Keeney, R.L. \& H. Raiffa (1976), "Decisions with Multiple Objectives." Wiley, New York. (Second edition 1993, Cambridge University Press, Cambridge). 
Miyamoto, J. \& P.P. Wakker (1996), "Multiattribute Utility Theory without Expected Utility Foundations," Operations Research 44 313-326.

Nakamura, Y. (1990), "Subjective Expected Utility with Non-additive Probabilities on Finite State Spaces," Journal of Economic Theory 51 346-366.

Nakamura, Y. (1996), "Sumex Utility Functions," Mathematical Social Sciences 31 $39-47$.

Saha, A. (1993), "Expo-Power Utility: A 'Flexible' Form for Absolute and Relative Risk Aversion," American Journal of Agricultural Economics 75 905-913.

Savage, L.J. (1954), "The Foundations of Statistics," Wiley, New York, Second edition 1972, Dover, New York.

Schmeidler, D. (1989), "Subjective Probability and Expected Utility without Additivity," Econometrica 57 571-587.

Smidts, A. (1997), "The Relationship Between Risk Attitudes and Strength of Preference: A Test of Intrinsic Risk Attitude," Management Science 43 357-370.

Tversky, A. \& D. Kahneman (1992), "Advances in Prospect Theory: Cumulative Representation of Uncertainty," Journal of Risk and Uncertainty 5 297-323.

von Neumann, J. \& O. Morgenstern (1944, 1947, 1953), "Theory of Games and Economic Behavior." Princeton University Press, Princeton NJ.

Wakker, P.P. (1984), "Cardinal Coordinate Independence for Expected Utility," Journal of Mathematical Psychology 28 110-117.

Wakker, P.P. (1989), “Additive Representations of Preferences, A New Foundation of Decision Analysis," Kluwer Academic Publishers, Dordrecht, The Netherlands.

Wakker, P.P. (1993), “Additive Representations on Rank-Ordered Sets II. The Topological Approach," Journal of Mathematical Economics 22 1-26. 
Wakker, P.P. \& H. Zank (1997) "A Simple Axiomatization of Rank-Dependent Utility and Cumulative Prospect Theory with Constant Proportional Risk Aversion," Research Memorandum RM/98/003, Maastricht University, The Netherlands. 